\title{
Synaptic Defects in a Drosophila Model of Congenital Muscular Dystrophy
}

\author{
Yogesh P. Wairkar, ${ }^{1}$ Lee G. Fradkin, ${ }^{2}$ Jasprina N. Noordermeer, ${ }^{2}$ and Aaron DiAntonio ${ }^{1}$ \\ ${ }^{1}$ Department of Molecular Biology and Pharmacology, Washington University Medical School, St. Louis, Missouri 63110, and ${ }^{2}$ Laboratory of Developmental \\ Neurobiology, Department of Molecular and Cell Biology, Leiden University Medical Center, 2300 RC Leiden, The Netherlands
}

The congenital muscular dystrophies present in infancy with muscle weakness and are often associated with mental retardation. Many of these inherited disorders share a common etiology: defective $O$-glycosylation of $\alpha$-dystroglycan, a component of the dystrophin complex. Protein-O-mannosyl transferase 1 (POMT1) is the first enzyme required for the glycosylation of $\alpha$-dystroglycan, and mutations in the POMT1 gene can lead to both Walker-Warburg syndrome (WWS) and limb girdle muscular dystrophy type 2K (LGMD2K). WWS is associated with severe mental retardation and major structural abnormalities in the brain; however, LGMD2K patients display a more mild retardation with no obvious structural defects in the brain. In a screen for synaptic mutants in Drosophila, we identified mutations in the Drosophila ortholog of POMT1, dPOMT1. Because synaptic defects are a plausible cause of mental retardation, we investigated the molecular and physiological defects associated with loss of $d P O M T 1$ in Drosophila. In $D P O M T 1$ mutants, there is a decrease in the efficacy of synaptic transmission and a change in the subunit composition of the postsynaptic glutamate receptors at the neuromuscular junction. We demonstrate that dPOMT1 is required to glycosylate the Drosophila dystroglycan ortholog Dg in vivo, and that this is the likely cause of these synaptic defects because (1) mutations in $D g$ lead to similar synaptic defects and (2) genetic interaction studies suggest that $d P O M T 1$ and $D g$ function in the same pathway. These results are consistent with the model that ${ }^{2} O M T 1$-dependent glycosylation of Dg is necessary for proper synaptic function and raise the possibility that similar synaptic defects occur in the congenital muscular dystrophies.

Key words: Drosophila; NMJ; POMT1; dystroglycan; glutamate receptors; muscular dystrophy

\section{Introduction}

The congenital muscular dystrophies (CMDs) are a devastating group of inherited neuromuscular disorders that are characterized by the onset of muscle weakness early during infancy. A number of genetically distinct CMDs share an underlying molecular cause: defects in protein $\mathrm{O}$-mannosylation (Michele et al., 2002; Grewal and Hewitt, 2003; Godfrey et al., 2007). Mutations in genes that encode putative glycosylation enzymes have been identified in a number of clinically distinct CMDs and are associated with hypoglycosylation of $\alpha$-dystroglycan ( $\alpha$-DG), a component of the dystrophin complex that links the cytoskeleton to the extracellular matrix (Haliloglu and Topaloglu, 2004). Mutations in protein $O$-mannosyl transferase 1 (POMT1), the first enzyme necessary for $O$-mannosylation, are present in some patients with two CMD variants, Walker-Warburg syndrome (WWS) and limb girdle muscular dystrophy type 2K (LGMD2K)

\footnotetext{
Received Sept. 25, 2007; accepted Feb. 29, 2008.

This work was supported by grants from the Muscular Dystrophy Association and National Institutes of Health (NS043171) to A.D. and by a Pioneer grant from the Netherlands Organization for Scientific Research to J.N.N. We thank Hannele Ruohola-Baker for the dystroglycan alleles and Martina Schneider for anti-dystroglycan (Dg ${ }^{\text {pep }}$ antibodies. We thank the Bloomington Stock Center (Bloomington, IN) for the flies used in this study. We thank James Skeath, E. J. Brace, Richard Daniels, Catherine Collins, and N. Rajalaxmi for their helpful comments on this manuscript. We also thank Xiaolu Sun and Anja de Jong for excellent technical assistance.

Correspondence should be addressed to Aaron DiAntonio, Department of Molecular Biology and Pharmacology, Washington University Medical School, 660 S. Euclid Avenue, Campus Box 8103, St. Louis, M0 63110. E-mail: diantonio@wustl.edu.

DOI:10.1523/JNEUROSCI.0478-08.2008

Copyright $\odot 2008$ Society for Neuroscience $\quad 0270-6474 / 08 / 283781-09 \$ 15.00 / 0$
}

(Balci et al., 2005; D’Amico et al., 2006; van Reeuwijk et al., 2006). Both WWS and LGMD2 are associated with mental retardation. In WWS, the mental retardation is severe and is likely caused by structural abnormalities secondary to cell migration defects (Beltran-Valero de Bernabe et al., 2002). In LGMD2, the mental retardation can be mild and is not associated with structural abnormalities in the brain, suggesting that POMT1 deficiency may lead to more subtle defects in neuronal structure or function (Balci et al., 2005).

In a screen for synaptic abnormalities at the Drosophila neuromuscular junction (NMJ), we identified mutations in the Drosophila homolog of POMT1, rotated abdomen $(r t)$, hereafter referred to as APOMT1. dPOMT1 and its enzymatic partner dPOMT2 are highly homologous to the human hPOMT1 and hPOMT2, respectively, and both are required for proper glycosylation of Dg in SF21 cells (Ichimiya et al., 2004). It remains unclear, however, whether they are required for glycosylation of

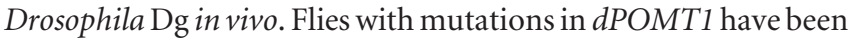
described previously as having a rotated or twisted abdomen that disrupts mating (Martin-Blanco and Garcia-Bellido, 1996; Ichimiya et al., 2004). Because mutations in human POMT1 can lead to mental retardation of unknown etiology, and because synaptic defects possibly result in mental retardation, we performed a detailed analysis of molecular and physiological synaptic defects associated with this Drosophila model of CMD.

The Drosophila NMJ is a well established system for studying the structure and function of glutamatergic synapses (Collins and 
DiAntonio, 2007). The postsynaptic glutamate receptor at the $\mathrm{NMJ}$ is comprised of three essential subunits and either of two nonessential subunits, DGluRIIA and DGluRIIB, which confer distinct physiological and pharmacological properties to the receptor (DiAntonio, 2006). In a large-scale screen, we identified dPOMT1 as a gene that, when mutated, leads to a selective reduction in the levels of DGluRIIB. In addition, synaptic strength is impaired in $A P O M T 1$ mutants because of a decrease in the number of synaptic vesicles released from the presynaptic motoneuron. This synaptic requirement for POMT1 is likely a result of its role in glycosylating Dg, because we show that (1) dPOMT1 is required to glycosylate $\mathrm{Dg}$ in vivo, (2) loss-of-function mutations in $D g$ phenocopy dPOMT1 mutations, and (3) dPOMT1 and Dg genetically interact. These results are consistent with the model that POMT1-dependent glycosylation of Dg is necessary for proper synaptic function and development at the Drosophila NMJ and raise the possibility that similar synaptic defects occur in the human CMDs.

\section{Materials and Methods}

Fly stocks. Flies were raised and maintained at $25^{\circ} \mathrm{C}$ on standard fly media. Wild-type (WT) flies were Canton S (CS) outcrossed to $\mathrm{w}^{-}$, elav-Gal4 (Yao and White, 1994), G7-Gal4 (Zhang et al., 2001), actin-Gal4 (Krasnow et al., 1989), or C142-Gal4 (de Jong et al., 2005) flies. The P-element collection (Bellen et al., 2004), including $r t^{P}$ line (the allele/gene is referred to as APOMT1) (Martin-Blanco and Garcia-Bellido, 1996), deficiency lines for $d P O M T 1$ [ $d f(3 L) E D 4470]$ and dystroglycan [Df(2)JP6 (Deng et al., 2003) and $D f(2 R) E D 2457$ ], and a P-element that is inserted in the dystroglycan gene (referred to as $\left.d g^{P}\right)(\mathrm{PBac}\{\mathrm{RB}\}$ Dge01554) were obtained from Bloomington Stock Center (Bloomington, IN). $\mathrm{Dg}^{323}$ and $D g^{248}$ were kindly provided by Dr. Hannele Ruohola-Baker (University of Washington, Seattle, WA). dPOMT1 transgenic lines were made by cloning the cDNA RE38203 (Ichimiya et al., 2004) into the pUAST vector (Brand and Perrimon, 1993).

Immunohistochemistry. Larvae were dissected and stained as described (Marrus et al., 2004). In brief, wandering third instar larvae were dissected in cold PBS and fixed for 5-10 min in Bouin's fixative and washed three times with PBS $+0.1 \%$ Triton X-100. Primary antibodies were used in the following dilutions: mouse $\alpha$-DGluRIIA (1:100); mouse $\alpha$-BRP (1:250) (Wagh et al., 2006) obtained from the Developmental Studies Hybridoma Bank, developed under the auspices of the National Institute of Child Health and Human Development and maintained by the Department of Biological Sciences of the University of Iowa (Iowa City, IA); rabbit $\alpha$-DGluRIII (1:2500); rabbit $\alpha$-DGluRIIB (1:2500) (Marrus et al., 2004); $\alpha$-dys- $\mathrm{CO}_{2} \mathrm{H}$ (1:2500) (van der Plas et al., 2006), and Cy3- or Cy5-conjugated goat $\alpha$-HRP (Jackson ImmunoResearch, West Grove, PA). Goat Cy5-, Cy3-, and FITC-conjugated secondary antibodies against mouse and rabbit IgG were used at 1:1000 and were obtained from Jackson ImmunoResearch.

Western blots. Wandering third instar larvae were dissected in ice-cold homogenization buffer (67 mm Tris- $\mathrm{HCl}, \mathrm{pH} 8.0,67 \mathrm{~mm} \mathrm{NaCl}, 2 \mathrm{M}$ urea, $1 \mathrm{~mm}$ EDTA, and $1.3 \%$ SDS) and stored immediately on dry ice. The samples were run on 6\% SDS-PAGE gels according to standard procedures. Rabbit $\alpha$-Dg ${ }^{\text {pep }}$ was used at 1:1000 (Schneider et al., 2006); mouse $\alpha$ - $\beta$-tubulin antibody (E7), obtained from the Developmental Studies Hybridoma Bank, developed under the auspices of the National Institute of Child Health and Human Development and maintained by the Department of Biological Sciences of the University of Iowa, was used at 1:100. HRP-conjugated goat $\alpha$-rabbit secondary antibody (Jackson ImmunoResearch) was used at 1:10,000.

Imaging and analysis. Imaging and analysis was done as described previously (Viquez et al., 2006) with the following minor differences. The average intensity function in MetaMorph was used to measure the intensity of the $\mathrm{NMJ}$ at muscle 4 . The experimenter was blinded to genotypes during both imaging and analysis. Statistical analysis was performed using one-way ANOVA for comparison of samples within an experimental group. $n$ for each condition is described in the figures. All histograms and measurements are shown as mean \pm SEM. Bruchpilot (BRP) puncta were manually counted at MN4b synapses on muscle 4 . While counting, we made sure that each of the BRP puncta had a DGluRIII puncta apposing it. Images for $\alpha$-dystrophin staining were acquired using a Zeiss (Thornwood, NY) digital camera attached to a Zeiss Axioplan microscope.

Electrophysiology. Intracellular electrophysiological recordings were done as reported previously (Marrus and DiAntonio, 2004). Briefly, wandering third-instar larvae were dissected in $0 \mathrm{~mm} \mathrm{Ca}^{2+}$ Stewart saline (HL3) (Stewart et al., 1994). Both spontaneous miniature excitatory junction potentials (mEJPs) and evoked potentials (EJPs) were then recorded $0.45 \mathrm{~mm}$ HL3. At least 60 consecutive miniature events were measured per cell using MiniAnalysis (Synaptosoft, Decatur, GA) and averaged to determine mean mEJP. Events with a slow rise time were rejected as likely artifacts arising from neighboring electrically coupled muscle cells. To record evoked EJPs, segmental nerves were cut and suctioned into a stimulating electrode, in which they received a brief depolarizing pulse. Quantal content was estimated by dividing the mean EJP by the mean $\mathrm{mEJP}(\mathrm{EJP} / \mathrm{mEJP})$. Cells across all genotypes had similar mean input resistances and resting potentials. Quantal size was not significantly different among any of the genotypes compared with wild-type controls. Statistical analysis was performed using one-way ANOVA to compare samples in an experimental group. Paired-pulse facilitation of the EJPs was assayed in $0.4 \mathrm{~mm} \mathrm{Ca}^{2+}$ with an interpulse interval of $50 \mathrm{~ms}$. Mean EJPs of the first and the fifth pulses were used for calculating the facilitation index/ratio. For electrotonic stimulation, recordings were performed as described by Song et al. (2002). Briefly, the stimulation electrode was kept as near as possible to the muscle, and the recordings were performed in $1 \mu \mathrm{M}$ TTX. EJCs were recorded as described by Marrus et al. (2004) in $2.0 \mathrm{~mm} \mathrm{Ca}^{2+}$ (Marrus and DiAntonio, 2004). Only recordings with holding currents $<1.0 \mathrm{nA}$ were analyzed.

\section{Results}

\section{Mutations in Drosophila POMT1 affect glutamate receptor subunit composition at the neuromuscular junction}

Clustering of neurotransmitter receptors opposite active zones is essential for effective synaptic transmission. At the Drosophila NMJ, postsynaptic glutamate receptors are comprised of three essential subunits (DGluRIII, DGluRIID, and DGluRIIE), as well as either of two nonessential subunits (DGluRIIA or DGluRIIB) (Marrus et al., 2004; Featherstone et al., 2005; Qin et al., 2005). These two classes of receptors differ in their localization, physiological properties, and modulation by second messenger systems (Petersen et al., 1997; Davis et al., 1998; DiAntonio et al., 1999; Chen et al., 2005). To investigate molecular mechanisms that control the clustering and subunit composition of synaptic glutamate receptors, we have screened a large collection of Drosophila mutants for changes in glutamate receptor localization.

We stained for glutamate receptors at the NMJs from $\sim 1500$ lines carrying unique insertions of P-element transposons in or near genes in the third chromosome (Bellen et al., 2004). Among this group, we identified a P-element insertion, $r t^{P}$, which has a decrease in the levels of synaptic DGluRIIB with no detectable change in the levels of DGluRIIA. The $r t^{P}$ transposon insertion was previously identified as an allele of $r t$ (referred to here as dPOMT1), the Drosophila ortholog of the congenital muscular dystrophy gene dPOMT1 (Martin-Blanco and Garcia-Bellido, 1996; Jurado et al., 1999). A second P-element insertion within the $d P O M T 1$ locus, $\mathrm{P}\{$ SUPor-P $\} \mathrm{rt}^{\mathrm{KG} 04772}$, shows a similar phenotype (data not shown). We assayed glutamate receptor localization quantitatively in a transheterozygote between the $r t^{P}$ insertion and a genetically unrelated deficiency that deletes dPOMT1 and surrounding genes (Fig. $1 A, B$ ). This transheterozygote also displays a selective reduction in DGluRIIB levels at the synapse (Fig. $1 A, B)(p<0.001)$, demonstrating that this phenotype is caused by the loss of $d P O M T 1$ and not by a secondsite mutation elsewhere on the $r t^{P}$ chromosome. To investigate 
A.
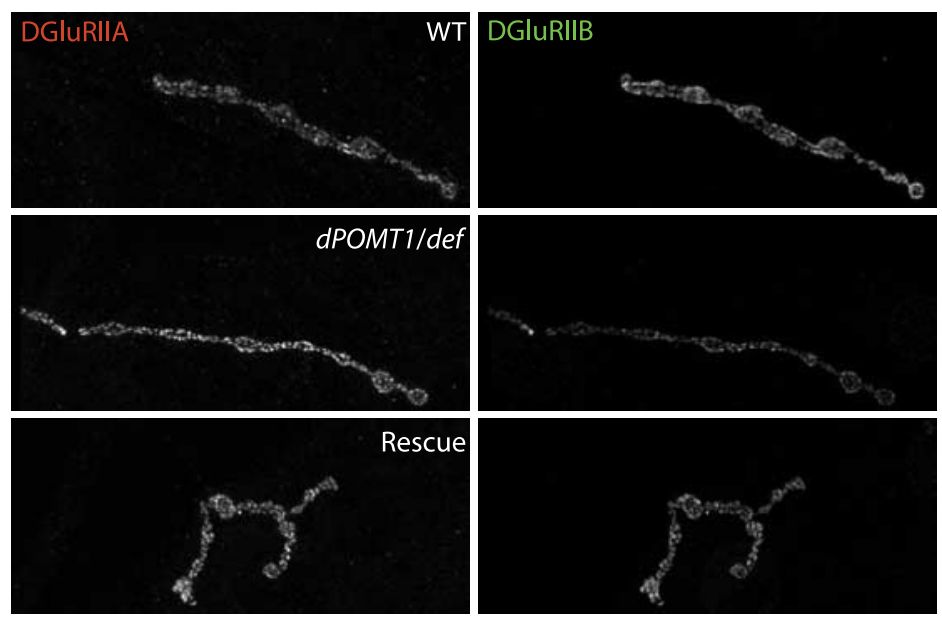
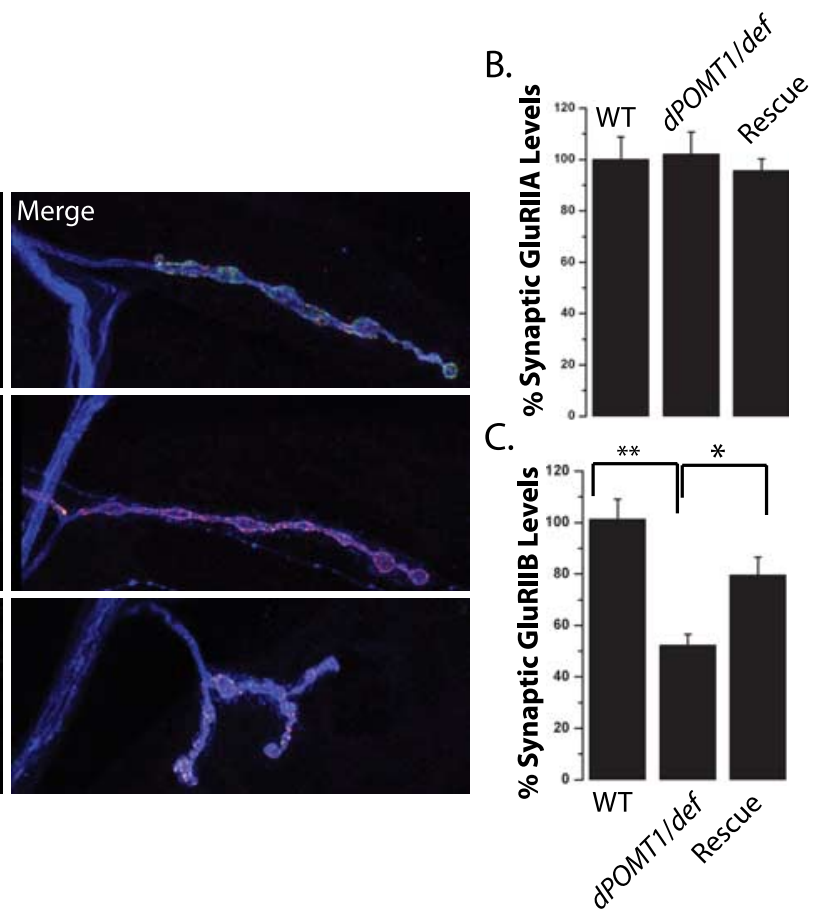

Figure 1. Mutations in XPOMT1 alter glutamate receptor subunit composition at the neuromuscular junction. A, Confocal images of larval NMJs stained with antibodies against DGluRIIA (red), DGLURIIB (green), and HRP (blue). The genotypes of the larvae are as follows: WT (CS crossed with (142-Gal4), the dPOMT1 mutant (dPOMT1/def), and the dPOMT1 mutant rescued with a dPOMT1 transgene [C142-GAL4/UAS-dPOMT1; rt $/ d f(3 L)$ ED4470]. $\boldsymbol{B}, \boldsymbol{C}$, Histograms show the mean staining intensity for DGluRIIA $(\boldsymbol{B})$ and DGluRIIB $(\boldsymbol{C})$ at the NMJ as a percentage of WT levels. Levels of DGluRIIB in the dPOMT1 mutant are significantly lower than in WT $\left({ }^{*} p<0.01\right)$, and DGluRIIB levels increase at the NMJ after rescue $\left({ }^{*} p<0.01\right) . n=10$ for all genotypes. Error bars represent SEM.

whether the $r t^{P}$ allele expresses $d P O M T 1$ transcript, we performed reverse transcription-PCR from WT and $r t^{p} /$ $d f(3 L) E D 4470$ ( $d P O M T 1 / d e f)$ flies. The $d P O M T 1$ transcript is present in wild-type tissue, but is not detectable in the $d P O M T 1 /$ defline (supplemental Fig. $1 C$, available at www.jneurosci.org as supplemental material), suggesting that the $r t^{P}$ (dPOMT1) mutant is either a severe hypomorph or a null. In this $d P O M T 1$ mutant, other aspects of synapse morphology are unaffected. There is no significant difference in the levels or distribution of the synaptic vesicle protein DVGLUT (Daniels et al., 2004) or the postsynaptic scaffolding protein DLG (Lahey et al., 1994) (supplemental Fig. $1 A$, available at www.jneurosci.org as supplemental material). In addition, both the number and size of synaptic boutons are unchanged in the mutant (supplemental Fig. $1 A, B$, available at www.jneurosci.org as supplemental material). Hence, the selective reduction of synaptic DGluRIIB is the most prominent anatomical defect in the $d P O M T 1$ mutant.

To investigate where POMT1 functions, we used the Gal4/ UAS system (Brand and Perrimon, 1993) to express transgenic dPOMT1 in the $r t^{P}$ (dPOMT1)/def mutant background. We generated a UAS- $d P O M T 1$ transgene that rescues the rotated abdomen phenotype of $r t^{P}$ (dPOMT1) when ubiquitously expressed under the control of actin-Gal4 (Krasnow et al., 1989) (supplemental Fig. 2, available at www.jneurosci.org as supplemental material). Hence, our UAS-dPOMT1 transgene is functional, and the rotated abdomen phenotype is attributable to loss of dPOMT1. In an attempt to rescue the selective loss of DGluRIIB, we expressed $A P O M T 1$ in the presynaptic neuron with elav-Gal4 (Yao and White, 1994), the postsynaptic muscle with G7-Gal4 (Zhang et al., 2001), or simultaneously on both sides of synapse using C142-Gal4 (de Jong et al., 2005). The selective expression of dPOMT1 in either nerve or muscle fails to rescue the DGluRIIB phenotype (supplemental Fig. $3 A, B$, available at www.jneurosci.org as supplemental material). However, when $d P O M T 1$ is expressed in neurons and muscles with C142-Gal4, there is a significant increase in the levels of DGluRIIB at the synapse (Fig. 1C). The mutant is also rescued by the ubiquitous expression of dPOMT1 driven by actinGal4 $(\sim 40 \% ; p<0.01 ; n=10)$. The rescue of $r t^{P}(d P O M T 1)$ by the transgenic expression of $\mathrm{dPOMT1}$ demonstrates that the loss of dPOMT1 function is responsible for decrease in DGluRIIB at the synapse. Rescue of morphology was not complete, however, suggesting that the levels or timing of $A P O M T 1$ expression may be critical for its full function. In addition, the failure to rescue with either presynaptic or postsynaptic expression suggests that DPOMT1 is required on both sides of the synapse.

\section{dPOMT1 promotes neurotransmitter release by increasing} probability of release

Changes in the subunit composition of the Drosophila glutamate receptor complex can change the function and structure of the NMJ (DiAntonio et al., 1999; Sigrist et al., 2002). DGluRIIA is the predominant subunit at a wild-type synapse, and the genetic deletion of DGluRIIB in the presence of wild-type levels of DGluRIIA does not lead to a change in quantal size, a measure of the postsynaptic response to a single vesicle (DiAntonio et al., 1999). As such, the selective reduction in DGluRIIB would not be predicted to decrease quantal size. To investigate synaptic function in the $d P O M T 1 \mathrm{mu}-$ tant, we performed intracellular recordings from muscle 6 of seg-

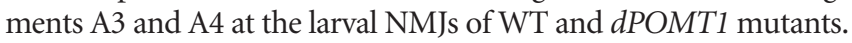
We did not observe a significant decrease in the amplitude of miniature excitatory junctional potentials (mEJP: WT, $0.79 \pm 0.02 \mathrm{mV}$; dPOMT1, $0.77 \pm 0.03 \mathrm{mV} ; n=9 ; p=0.4$ ) or frequency of mEJP (frequency: WT, $2.5 \pm 0.9 \mathrm{~Hz}$; dPOMT1, $3.1 \pm 1.1 \mathrm{~Hz} ; n=9 ; p=$ $0.3)$ (Fig. 2A). There is, however, a dramatic decrease in evoked synaptic transmission. Indeed, the EJP amplitude is decreased nearly threefold in the $A P O M T 1$ mutant (Fig. $2 B$ ). Because the response to 
a single vesicle is unchanged, the decrease in evoked release indicates that the nerve is releasing fewer synaptic vesicles. Indeed, estimates of quantal content based on the direct method (EJP/mEJP) show an approximately threefold reduction in the number of released vesicles (Fig. 2C). As with the change in DGluRIIB levels, expression of $d P O M T 1$ in neither the nerve nor muscle was able to rescue the defect in synaptic release (data not shown). However, expression of $d P O M T 1$ using $\mathrm{C} 142-\mathrm{Gal} 4$ restores transmitter release to wild-type levels (Fig. 2C). Expression of dPOMT1 from the ubiquitous actin-Gal4 driver also rescues quantal content $(\sim 50 \%$ increase; $p<0.001 ; n=9)$. Hence, the loss of dPOMT1 is responsible for the reduced quantal content in the $r t^{P}(d P O M T 1)$ mutant, and therefore $A P O M T 1$ is required for normal synaptic transmission at the Drosophila NMJ.

Decreased transmitter release could be caused by defects in the generation or propagation of action potentials to the synapse. To test this hypothesis, we bypassed action potential-stimulated release by direct electrotonic stimulation of the nerve in $1 \mu \mathrm{M}$ TTX, which suppresses action potentials (Song et al., 2002). If reduced transmitter release was a result of action potential defects, then direct stimulation should rescue the phenotype and lead to normal transmitter release. If the defect is downstream of the action potential, then electrotonic stimulation should still lead to defective transmitter release. With electrotonic stimulation in the presence of TTX, there is a significant decrease in evoked transmitter release in the $r^{P}(d P O M T 1) / d e f$ mutant when compared with wild type (Fig. 2D). This difference in quantal content is similar to that observed after action potentialstimulated release. Thus, defective action potentials do not explain the reduction in quantal content in $\mathrm{APOMT1}$ mutants.

Why is quantal content decreased in the $A P O M T 1$ mutant? Reduced quantal content could be caused by a decrease in the number of release sites $(n)$ or by a decrease in the probability that a synaptic vesicle will be released from a release site $(p)$. To investigate the mechanism responsible for the decrease in quantal content in the $A P O M T 1$ mutants, we have assessed both $n$ and $p$. Active zones are the sites of neurotransmitter release, and in Drosophila individual release sites can be observed by staining for Bruchpilot, the Drosophila homolog of the active zone protein CAST (Wagh et al., 2006). Each active zone is apposed to a cluster of glutamate receptors (Marrus and DiAntonio, 2004), and a functional release site includes both elements of this synaptic dyad. We stained the NMJ of wild type and $A P O M T 1$ mutants with $\alpha$-Bruchpilot and an antibody to the essential glutamate receptor subunit DGluRIII and counted the number of release sites (Collins and DiAntonio, 2007; Pack-Chung et al., 2007; Dickman et al., 2008) (Fig. 3A). There is no significant difference in active zone number in the $d P O M T 1$ mutant (WT, $254 \pm 16$; dPOMT1/def, $252 \pm 18 ; n=9$ ). In addition, almost all active zones are apposed to glutamate receptors at both wild-type and mutant NMJs; thus, there is no evidence for postsynaptically silent synapses. The frequency of mEJPs in $d P O M T 1 /$ def is also not
B.
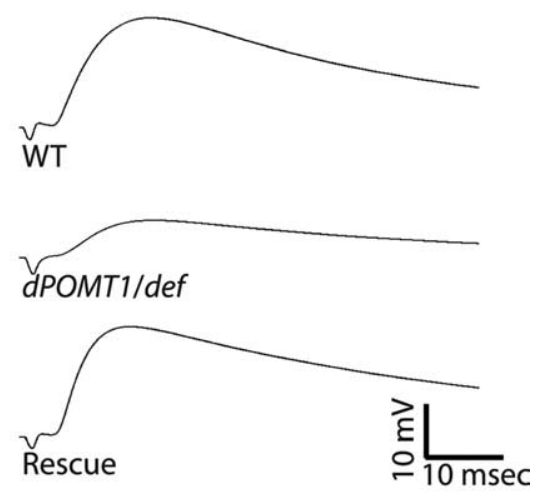

D.

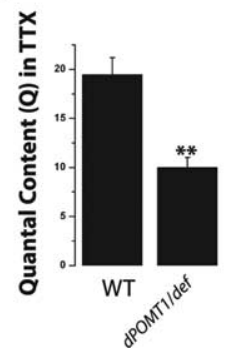

significantly different from that of WT. Together, these data indicate that a change in the number of release sites is unlikely to explain the defects in transmitter release in the $\mathrm{APOMT1}$ mutant.

The probability $(p)$ that a synaptic vesicle will be released after an action potential is another key determinant of synaptic release. To investigate potential changes in release probability, we measured short-term facilitation, which varies inversely with $p$. When a nerve is repeatedly stimulated with a short interpulse interval, the subsequent evoked events are often larger because of residual calcium in the presynaptic terminal (Zucker and Regehr, 2002). When probability of release is low, such short-term facilitation is more pronounced. After a short train of stimuli (see Materials and Methods for details), we measured the amplitude of each EJP and calculated the facilitation index as the ratio of the fifth to the first EJP. Although the wild-type NMJ facilitates, the $A P O M T 1$ mutant NMJ has greatly enhanced facilitation, leading to an approximately onefold increase in the degree of facilitation (Fig. $3 B, C$ ), consistent with a decrease release probability. To test this hypothesis directly, we recorded evoked junctional currents (EJCs) from WT and $d P O M T 1$ mutants in presence of high external calcium $\left(2.0 \mathrm{mM} \mathrm{Ca}^{2+}\right)$. At this calcium concentration, the probability of release is very high, and transmitter release is near saturation. High calcium should rescue defects in probability of release, but would not rescue a reduction in the number of release sites. The dPOMT1/def mutants display wild-type EJC amplitudes when recorded in high calcium (WT, $66 \pm 3.0 \mathrm{pA}$; dPOMT1/def, $57 \pm 3.7 \mathrm{pA} ; n=10 ; p=0.3$ ) (Fig. $3 D$ ), indicating that the mutant defect likely lies at the level of release probability. The rescue of transmitter release by high calcium, the large increase in facilitation at low calcium, and the lack of change in anatomically identifiable release sites in the mutant indicates that $\mathrm{dPOMT} 1$ promotes transmitter release by enhancing release probability. 
A.
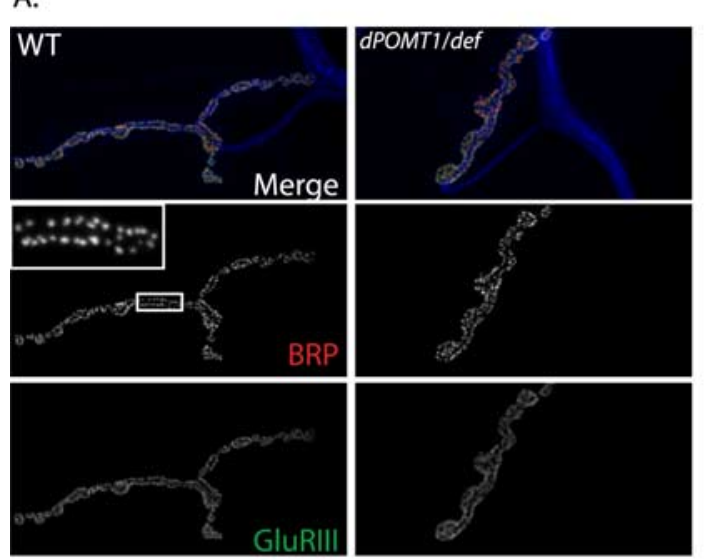

B.

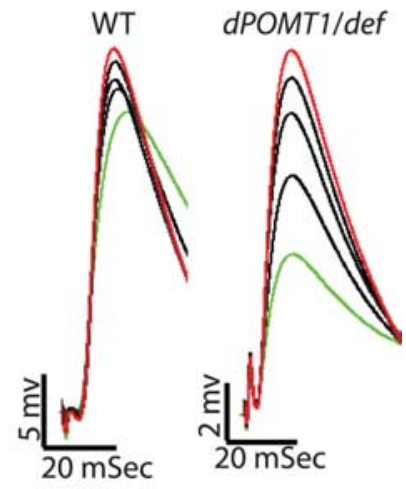

C.

D.
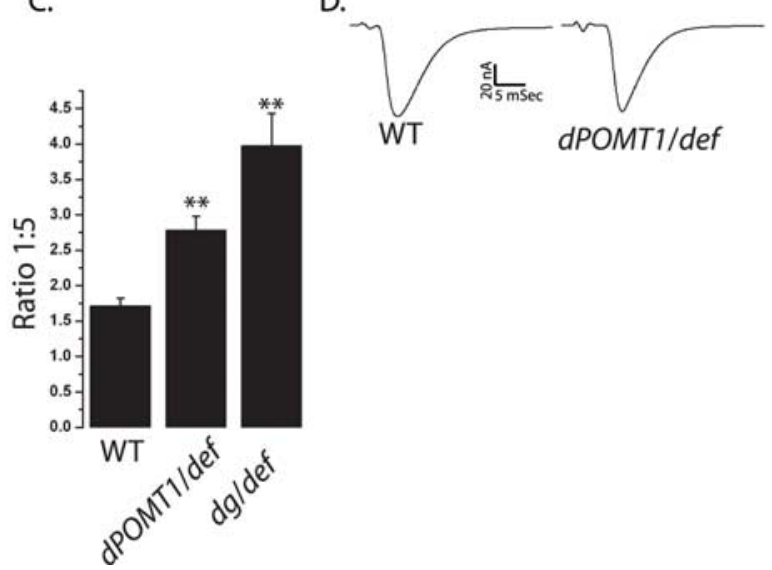

Figure 3. Probability of release is decreased in DPOMT1 mutants. $\boldsymbol{A}$, NMJs stained for active zones ( $\alpha$-BRP; red), glutamate receptor clusters ( $\alpha$-DGluRIII; green), and neuronal membrane ( $\alpha$-HRP; blue) from WT (CS crossed with $w^{-}$) and $d P O M T 1$ mutant (dPOMT1/def) larvae. Individual BRP puncta ( $\boldsymbol{A}$, inset) were counted from the entire NMJ $(n=9$ for both; $p=0.8)$. $\boldsymbol{B}$, First through fifth EJP sweeps in response to a train of stimuli in WT (CS crossed to $W^{-}$) and the dPOMT1 mutant (dPOMT1/def) larvae. C, Quantification of the facilitation index (FI) for WT and dPOMT1/def. $n$ for WT $=7$ and for $d P 0 M T 1 / d e f=9 ; p<0.001$. The dystroglycan mutant $\left(D g^{P} /\right.$ def $)$ shows an increase in the FI similar to that of $d P O M T 1 /$ def. $n=9 ;{ }^{* *} p<0.001$. FI was calculated by dividing the fifth pulse $(\boldsymbol{B}$, red) by the first pulse ( $\boldsymbol{B}$, green). Error bars represent SEM. $\boldsymbol{D}$, Sample EJC traces from WT and mutant (dPOMT1/def) larvae. $n=10 ; p=0.3$.

\section{dPOMT1 is required for the glycosylation of dystroglycan in vivo}

Because POMT1 is required to O-glycosylate proteins, the lack of glycosylation of a target protein is a likely explanation for the phenotype of $A P O M T 1$ mutants. In vertebrates, POMT1 glycosylates $\alpha$-dystroglycan, and the loss of POMT1 result in hypoglycosylation, and in some cases loss, of dystroglycan (Jimenez-Mallebrera et al., 2003; Akasaka-Manya et al., 2004; Kim et al., 2004). Because glycosylation is important for dystroglycan function, the congenital muscular dystrophies associated with mutations in POMT1 likely are caused by loss of dystroglycan function. In Drosophila, dPOMT1 can promote the glycosylation of the dystroglycan ortholog Dg in vitro (Ichimiya et al., 2004), but it is not known whether it is required in vivo. To investigate whether $\mathrm{APOMT1}$ mutants affect the glycosylation state of Dg, we performed a Western blot with protein extract from larval brain and body walls of wild type and $A P O M T 1$ mutants and probed for Dg with the previously characterized $\mathrm{dg}^{\mathrm{pep}}$ antibody (Schneider et al., 2006). This antibody recognizes two bands on a Western blot, one of $\sim 110 \mathrm{kDa}$, the predicted size for the unmodified protein, and another of $>200 \mathrm{kDa}$, which is thought to be the glycosylated form (Schneider et al., 2006). From the immunoblot, levels of the $110 \mathrm{kDa}$ gg band are not significantly different between wild-type and $d P O M T 1$ mutant larvae (Fig. $4 B)(p=0.3)$; however, the $>200 \mathrm{kDa} \mathrm{Dg}$ band is undetectable in the APOMT1 mutants (Fig. $4 A, C$ ). This loss of the putative glycosylated form of $\mathrm{Dg}$ is restored by the transgenic expression of dPOMT1. These data indicate that $\mathrm{Dg}$ is a likely target of dPOMT1 in the fly. In the absence of dPOMT1, we do not observe the highmolecular-weight glycosylated form of Dg; however, the levels of the nonglycosylated form are approximately normal. Hence, there are two changes to Dg protein in dPOMT1 mutants: total expression levels are reduced, and there is no apparent glycosylation. Either or both of these alterations may underlie the phenotypes we observe. If the nonglycosylated form of $\mathrm{Dg}$ can function, then overexpression of $D g$ should rescue defects caused by loss of $d P O M T 1$. However, we find that whereas overexpressing $D g$ in a wild-type background leads to no gross defects, overexpression of $D g$ in the $d P O M T 1$ mutant leads to lethality (data not shown). This suggests that glycosylation of Dg is essential for its function and that increased levels of the nonglycosylated Dg may be toxic.

Dystroglycan is a component of the dystrophin glycoprotein complex (Ervasti et al., 1990). We wished to assess whether loss of glycosylated Dg affects the levels and localization of dystrophin. Drosophila dystrophin localizes to both the neuromuscular junction and the muscle sarcomeres (van der Plas et al., 2006, 2007). In the APOMT1 mutant, dystrophin still localizes to these structures (Fig. 4D), so glycosylated Dg is not necessary for the localization of dystrophin, consistent with results from vertebrates (Cote et al., 2002). Although dystrophin is still present at the $\mathrm{NMJ}$ and sarcomeres, its levels are reduced. At the NMJ, there is an $\sim 20 \%$ reduction in dystrophin $(p<0.01)$, which is restored with transgenic $d P O M T 1$ rescue (Fig. $4 D, E$ ). A similar reduction is observed for sarcomeric dystrophin (data not shown), suggesting that the glycosylation of $\mathrm{Dg}$ promotes the stability of dystrophin.

\section{Dystroglycan and dPOMT1 mutants genetically interact}

Because Dg is abnormally processed in the $A P O M T 1$ mutant, the dPOMT1 phenotypes may be attributable to loss of Dg function. If this is the case, then $D g$ mutants may display phenotypes similar to those described above for $d P O M T 1$. We obtained two Dg mutants and two genetically unrelated deficiencies that delete $D g$ and adjacent genes. For both $D g$ mutants in combination with either deficiency, there is a significant decrease in the levels of the DGluRIIB subunit at the larval NMJ [WT, 100\% $\pm 6.8 \%$; $P B a c\{R B\} D g e 01554 /$ $\left.D f(2 R) E D 2457\left(g^{p} / d e f\right), 45 \% \pm 4.8 \% ; n=10 ; p<0.001\right]$, without a change in the levels of DGluRIIA (Fig. 5). Hence, loss of $D g$, like loss of $A P O M T 1$, leads to a change in glutamate receptor subunit composition. Electrophysiological studies also highlight the similarity in phenotype between $D g$ and $d P O M T 1$ mutants (Fig. $6 A, B$ ). Both $D g$ alleles have a marked reduction in the amplitude of evoked EJPs with no change in the amplitude of spontaneous mEJPs (WT, $0.96 \pm$ 
0.05; $D g^{P} /$ def, $\left.0.85 \pm 0.05 ; n=10 ; p=0.4\right)$. Hence, quantal content is dramatically reduced when $D g$ function is impaired (Fig. 6C). As with dPOMT1 mutants, this decrease in transmitter release is accompanied by an increase in short-term facilitation (Fig. $3 C$ ), consistent with a lowered probability of release. In summary, the synaptic phenotypes observed in APOMT1 mutants are also displayed by the $D g$ mutants.

The similarity in phenotypes between dPOMT1 and Dg mutants is consistent with the model that dPOMT1-dependent glycosylation of $\mathrm{Dg}$ is necessary to promote synaptic vesicle release and regulate glutamate receptor subunit composition. However, it is formally possible that the two genes function in parallel pathways. A powerful approach for identifying genes in the same pathway is to test for transheterozygous interactions. Heterozygous mutations (loss of one of two alleles) in either $d P O M T 1$ or $D g$ have no affect on either the levels of DGluRIIB or quantal content (Fig. 7C,D). If the genes function in parallel pathways, and neither pathway is impaired enough to show a phenotype, then we would predict that the transheterozygote, in which one allele of each gene is mutant, would also have no phenotype. However, if the genes function in the same pathway, then mutations in two steps of one pathway may enhance each other and lead to phenotype; this is what we observe. The transheterozygote between $d P O M T 1$ and $D g$ exhibits both a decrease in the levels of DGluRIIB at the NMJ (Fig. $7 A, C$ ) and a marked reduction in quantal content (Fig. $7 B, D$ ) without a decrease in mEJP amplitudes (Fig. $7 E$ ). This experiment demonstrates that $\triangle P O M T 1$ and $D g$ genetically interact and, in conjunction with the biochemical data, supports the model that the glycosylation of Dg by dPOMT1 is required for normal synaptic development and function at the Drosophila NMJ.

\section{Discussion}

POMT1 is required for the $O$-glycosylation of dystroglycan, and mutations in POMT1 can lead to two variants of congenital muscular dystrophy, WWS and LGMD2K (Balci et al., 2005; D’Amico et al., 2006; van Reeuwijk et al., 2006). Both diseases are associated with mental retardation; however, for the milder LGMD2 no apparent structural abnormalities are present in the brain that would explain the onset of mental retardation. In this study, we characterize a Drosophila model of POMT1 deficiency. We find that, as in vertebrates, Drosophila POMT1 is required for glycosylation of dystroglycan. In Drosophila, the inability to glycosylate dystroglycan, or the genetic disruption of dystroglycan, does not lead to gross structural abnormalities at the neuromuscular junction, but rather disrupts presynaptic glutamate release and alters the subunit composition of postsynaptic glutamate receptors. Similar synaptic changes at vertebrate central synapses are a potential cause of mental retardation in CMD patients.

\section{Glycosylation of dystroglycan by POMT1}

The glycosylation of dystroglycan is affected in many forms of CMD (Grewal and Hewitt, 2003; Haliloglu and Topaloglu, 2004).
C.

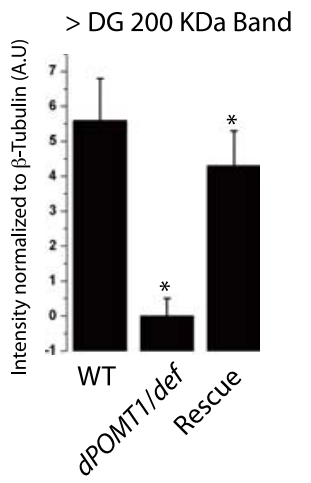

D.

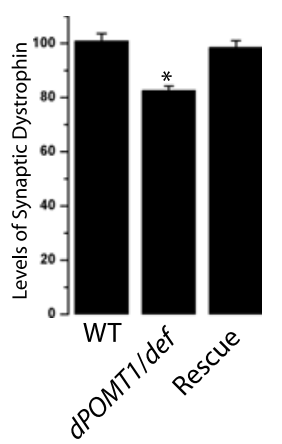

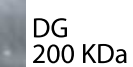

$\beta$-Tubulin

$110 \mathrm{KDa}$

$\beta$-Tubulin

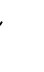

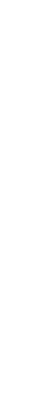

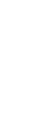

Figure 4. $\quad$ dPOMT1 is necessary for the glycosylation of dystroglycan in vivo. $A$, Western blot showing the glycosylated $>200$ kDa dystroglycan protein in WT (CS crossed to $W^{-}$), dPOMT1 mutant (dPOMT1/def), and dPOMT1 rescue [Act-GAL4/UAS-DPOMT1; of synaptic dystrophin levels in the same genotypes as in $\boldsymbol{A} . n=7$ (WT); $n=15$ (dPOMT1 mutant and rescue); ${ }^{*}<<0.01$. $\boldsymbol{E}$, Representative NMJs stained with $\alpha$-dystrophin antibody. The genotypes are identical to those in $\boldsymbol{A}$.

Glycosylation of dystroglycan is required for its binding to components of the extracellular matrix (Martin, 2007). In addition, loss of glycosylation can lead to a decrease in the levels of dystroglycan (Jimenez-Mallebrera et al., 2003; van Reeuwijk et al., 2005). Hence, glycosylation enzymes such as POMT1 may be required for both the activity and stability of dystroglycan. In Drosophila, dPOMT1 promotes the glycosylation of Dg in vitro, and the loss of $A P O M T 1$ in cultured Drosophila SF21 cells results in hypoglycosylation of Dg (Ichimiya et al., 2004). Here we demonstrate that dPOMT1 is required in vivo for the normal glycosylation of Dg. In the absence of dPOMT1, the total levels of dystroglycan are decreased, because the glycosylated band is lost with no commensurate increase in the nonglycosylated band. The failure to observe more nonglycosylated Dg suggests that glycosylation may be important for the stability of $\mathrm{Dg}$.

Drosophila perlecan binds to Dg that lacks the mucin-rich O-glycosylation domain (Schneider et al., 2006), so it is plausible that the nonglycosylated Dg could retain some function. The ability to manipulate Dg and DPOMT1 independently allowed us to test whether the decrease in total Dg was the major cause of the dPOMT1 phenotypes. We find that increasing the levels of dystroglycan in a $A P O M T 1$ mutant leads to lethality. This suggests that too much nonglycosylated Dg may be toxic, and is consistent with the model that glycosylation is required for $\mathrm{Dg}$ function.

\section{dPOMT1 and Dg are required for efficient} neurotransmitter release

Previous analysis of $A P O M T 1$ mutants demonstrated that loss of dPOMT1 leads to a rotated abdomen phenotype and disrupted muscle structure (Ichimiya et al., 2004; Lyalin et al., 2006; Haines et al., 2007). Our analysis adds a second major phenotype: a se- 

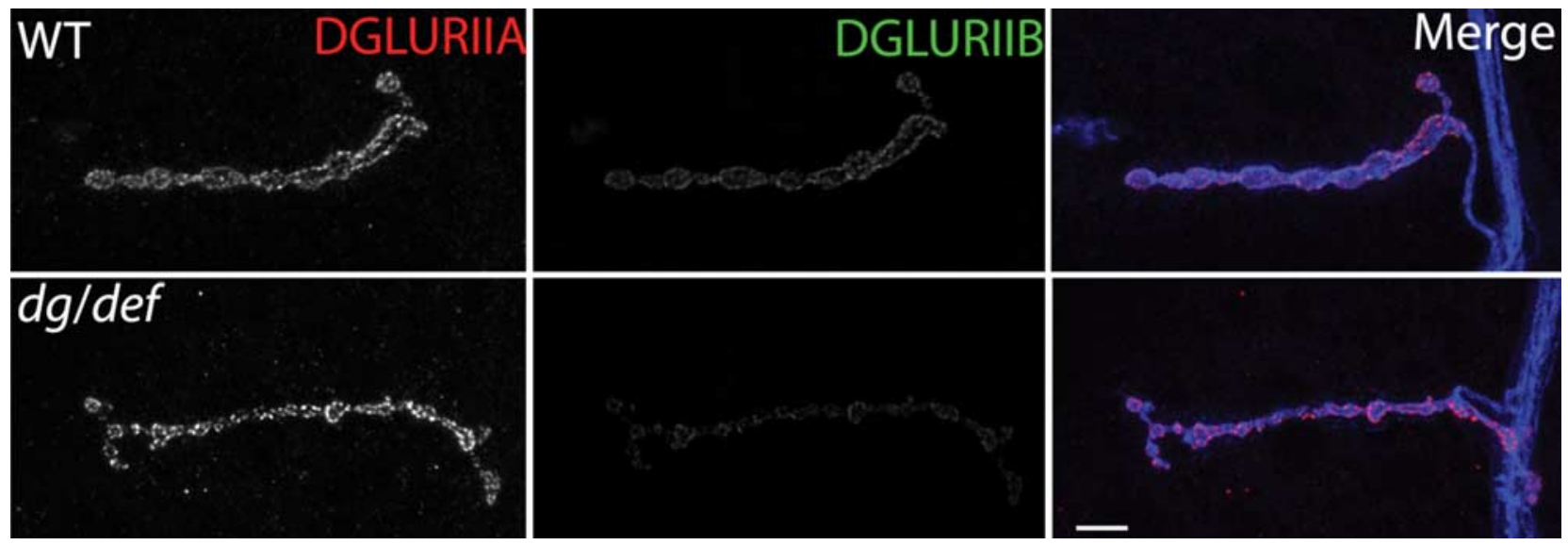

Figure 5. Mutants deficient in dystroglycan have altered glutamate receptor subunit composition. Sample larval NMJs from WT (CS crossed to $W^{-}$) and the dystroglycan mutant ( $\left.d g^{P} / d e f\right)$ stained with $\alpha$-DGluRIIA (red), $\alpha$-DGluRIIB (green), and $\alpha$-HRP (blue) antibodies. $n=10$ for all genotypes; ${ }^{* *} p<0.001$.

A.

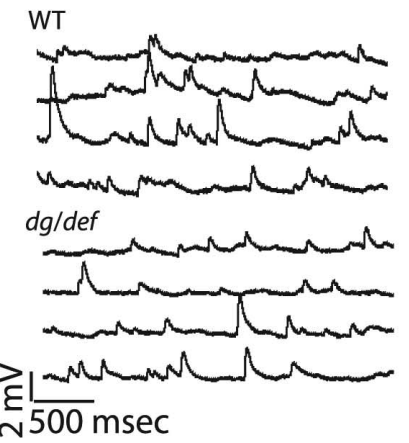

B.

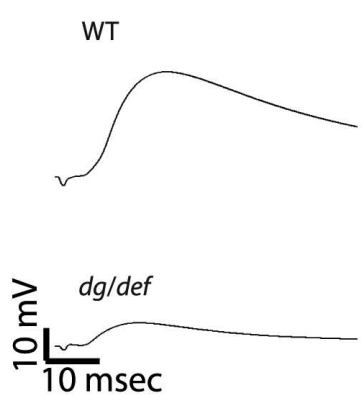

Figure 6. Quantal content is reduced in dystroglycan mutants. $\boldsymbol{A}$, Sample mEJP traces from WT and $D g^{P} /$ def larvae. Genotypes are the same as in Figure 5. $\boldsymbol{B}$, Sample EJP traces from WT and $D g^{P} /$ def larvae. C, Quantal content of WT and dystroglycan mutant $\left(d g^{p} / d e f\right)$ larvae. Quantal content was calculated by dividing the mean EJP amplitude by mean mEJP amplitude. $n=10$; ${ }^{* *} p<$ 0.001 .

vere impairment in the ability to release neurotransmitter. We investigated the mechanism underlying this synaptic phenotype. We detect no change in the number of anatomically defined neurotransmitter release sites $(n)$, suggesting that probability of release $(p)$ is impaired in the mutant. Consistent with this hypothesis, when transmitter is released in low calcium conditions, there is an increase in short-term facilitation, which usually varies inversely with release probability. In addition, high external calcium, which saturates release probability, rescues the defects in evoked transmitter release in the $A P O M T 1$ mutant. These data demonstrate that the defect in synaptic transmission in the dPOMT1 mutant is attributable to a reduction in release probability rather than a reduction in the number of release sites.

What might be the molecular cause of this decrease in probability of release? Our data suggest that the proximate cause is probably the loss of glycosylated dystroglycan. We find that mutations in $D g$ also have a decrease in $p$, and the strong genetic interactions between dPOMT1 and Dg heterozygotes are consistent with the genes working in the same pathway to promote transmitter release. Why then would the loss of glycosylated dystroglycan impair transmitter release? We do not know the answer, but speculate that dystroglycan, via its interactions with the extracellular matrix, is an important part of a transsynaptic complex that plays a structural and/or functional role at the synapse to promote normal synaptic function. Indeed, components of the extracellular matrix and dystrophin regulate synaptic function at the Drosophila NMJ (Johnson et al., 2006; van der
C.

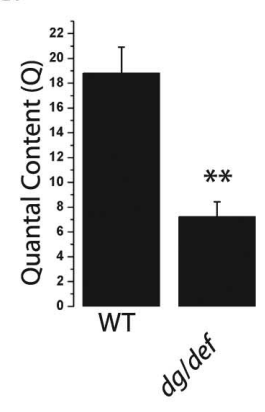

Plas et al., 2006). However, the reduced synaptic function in $A P O M T 1$ and $D g$ mutants is unlikely to be caused by the reduction in levels of postsynaptic dystrophin, because mutations in dystrophin lead to an increase, rather than decrease, in evoked transmitter release (van der Plas et al., 2006).

In which cells does glycosylated dystroglycan function to promote transmitter release? We have generated a functional dPOMT1 transgene whose ubiquitous expression rescues the rotated abdomen phenotype. We investigated the spatial requirement for dPOMT1 in synaptic function by driving the transgene at the NMJ using neuronal, muscle, ubiquitous, and neuronal/ muscle synaptic Gal4 driver lines. We find that the synaptic $d P O M T 1$ phenotypes are rescued only when the transgene is driven by either the neuronal/muscle C142-Gal4 or ubiquitous actin-Gal4 driver and not when it is expressed exclusively in the presynaptic or postsynaptic cell. Therefore, dPOMT1 may be required for glycosylating dystroglycan both in neurons and muscles to maintain the normal function of the NMJ. Dystroglycan is expressed in both muscles and brain in Drosophila (Dekkers et al., 2004; Shcherbata et al., 2007), and our results are consistent with the model that it functions in both neurons and muscles at the NMJ.

\section{Subunit-specific regulation of glutamate receptors by dPOMT1}

One of the intriguing findings of this study is the specific reduction in the DGluRIIB subunit of the glutamate receptor in $d P O M T 1$ and $d g$ mutants. At the Drosophila NMJ, postsynaptic glutamate receptors are comprised of three essential subunits as well as either of two nonessential subunits, DGluRIIA and DGluRIIB (for review, see DiAntonio 2006). Receptors with these alternate subunits are differentially localized opposite the terminals of distinct motoneurons that synapse with the same muscle cell, leading to the suggestion that presynaptic activity may shape glutamate receptor subunit composition (Marrus et al., 2004). Although extensive studies have been done in vertebrate AMPAtype receptor subunit composition and trafficking (Barry and Ziff, 2002; Malinow and Malenka, 2002; McGee and Bredt, 2003), mechanisms that describe such subunit-specific regulation of 
glutamate receptors are not well understood at the Drosophila NMJ. Recently, the actin/spectrin-binding protein Coracle was shown to regulate the subunit composition of glutamate receptors at the Drosophila NMJ. Mutations in Coracle lead to a specific loss in the DGluRIIA subunit, demonstrating that distinct molecular pathways can control subunit composition (Chen et al., 2005). Our results indicate that $d P O M T 1$ via $D g$ also regulates the subunit composition of glutamate receptors at the Drosophila NMJ. It is tempting to speculate that dystroglycan, which participates in clustering acetylcholine receptors in vertebrates (Cote et al., 1999; Grady et al., 2000), could be involved in the clustering of DGluRIIB subunit of glutamate receptors. However, it is also plausible that the changes in DGluRIIB levels are secondary to the changes in synaptic function and do not reflect a direct function of dystroglycan in receptor localization. These findings open a new path for understanding the molecular and/or activitydependent cues that control the localization of specific glutamate receptor subunits at the Drosophila NMJ.

\section{References}

Akasaka-Manya K, Manya H, Endo T (2004) Mutations of the POMT1 gene found in patients with Walker-Warburg syndrome lead to a defect of protein $\mathrm{O}$-mannosylation. Biochem Biophys Res Commun 325:75-79.

Balci B, Uyanik G, Dincer P, Gross C, Willer T, Talim B, Haliloglu G, Kale G, Hehr U, Winkler J, Topaloglu H (2005) An autosomal recessive limb girdle muscular dystrophy (LGMD2) with mild mental retardation is allelic to Walker-Warburg syndrome (WWS) caused by a mutation in the POMT1 gene. Neuromuscul Disord 15:271-275.

Barry MF, Ziff EB (2002) Receptor trafficking and the plasticity of excitatory synapses. Curr Opin Neurobiol 12:279-286.

Bellen HJ, Levis RW, Liao G, He Y, Carlson JW, Tsang G, Evans-Holm M, Hiesinger PR, Schulze KL, Rubin GM, Hoskins RA, Spradling AC (2004) The BDGP gene disruption project: single transposon insertions associated with $40 \%$ of Drosophila genes. Genetics 167:761-781.

Beltran-Valero de Bernabe D, Currier S, Steinbrecher A, Celli J, van Beusekom E, van der Zwaag B, Kayserili H, Merlini L, Chitayat D, Dobyns WB, Cormand B, Lehesjoki AE, Cruces J, Voit T, Walsh CA, van Bokhoven H, Brunner HG (2002) Mutations in the O-mannosyltransferase gene POMT1 give rise to the severe neuronal migration disorder WalkerWarburg syndrome. Am J Hum Genet 71:1033-1043.

Brand AH, Perrimon N (1993) Targeted gene expression as a means of altering cell fates and generating dominant phenotypes. Development 118:401-415.

Chen K, Merino C, Sigrist SJ, Featherstone DE (2005) The 4.1 protein coracle mediates subunit-selective anchoring of Drosophila glutamate receptors to the postsynaptic actin cytoskeleton. J Neurosci 25:6667-6675.

Collins CA, DiAntonio A (2007) Synaptic development: insights from Drosophila. Curr Opin Neurobiol 17:35-42.

Cote PD, Moukhles H, Lindenbaum M, Carbonetto S (1999) Chimaeric mice deficient in dystroglycans develop muscular dystrophy and have disrupted myoneural synapses. Nat Genet 23:338-342.

Cote PD, Moukhles H, Carbonetto S (2002) Dystroglycan is not required for localization of dystrophin, syntrophin, and neuronal nitric-oxide syn- thase at the sarcolemma but regulates integrin alpha 7B expression and caveolin-3 distribution. J Biol Chem 277:4672-4679.

D’Amico A, Tessa A, Bruno C, Petrini S, Biancheri R, Pane M, Pedemonte M, Ricci E, Falace A, Rossi A, Mercuri E, Santorelli FM, Bertini E (2006) Expanding the clinical spectrum of POMT1 phenotype. Neurology 66: 1564-1567; discussion 1461

Daniels RW, Collins CA, Gelfand MV, Dant J, Brooks ES, Krantz DE, DiAntonio A (2004) Increased expression of the Drosophila vesicular glutamate transporter leads to excess glutamate release and a compensatory decrease in quantal content. J Neurosci 24:10466-10474.

Davis GW, DiAntonio A, Petersen SA, Goodman CS (1998) Postsynaptic PKA controls quantal size and reveals a retrograde signal that regulates presynaptic transmitter release in Drosophila. Neuron 20:305-315.

de Jong S, Cavallo JA, Rios CD, Dworak HA, Sink H (2005) Target recognition and synaptogenesis by motor axons: responses to the sidestep protein. Int J Dev Neurosci 23:397-410.

Dekkers LC, van der Plas MC, van Loenen PB, den Dunnen JT, van Ommen GJ, Fradkin LG, Noordermeer JN (2004) Embryonic expression patterns of the Drosophila dystrophin-associated glycoprotein complex orthologs. Gene Expr Patterns 4:153-159.

Deng WM, Schneider M, Frock R, Castillejo-Lopez C, Gaman EA, Baumgartner S, Ruohola-Baker H (2003) Dystroglycan is required for polarizing the epithelial cells and the oocyte in Drosophila. Development 130:173-184.

DiAntonio A (2006) Glutamate receptors at the Drosophila neuromuscular junction. Int Rev Neurobiol 75:165-179.

DiAntonio A, Petersen SA, Heckmann M, Goodman CS (1999) Glutamate receptor expression regulates quantal size and quantal content at the Drosophila neuromuscular junction. J Neurosci 19:3023-3032.

Dickman DK, Kurshan PT, Schwarz TL (2008) Mutations in a Drosophila 
$\alpha 2 \delta$ voltage-gated calcium channel subunit reveal a crucial synaptic function. J Neurosci 28:31-38.

Ervasti JM, Ohlendieck K, Kahl SD, Gaver MG, Campbell KP (1990) Deficiency of a glycoprotein component of the dystrophin complex in dystrophic muscle. Nature 345:315-319.

Featherstone DE, Rushton E, Rohrbough J, Liebl F, Karr J, Sheng Q, Rodesch CK, Broadie K (2005) An essential Drosophila glutamate receptor subunit that functions in both central neuropil and neuromuscular junction. J Neurosci 25:3199-3208.

Godfrey C, Clement E, Mein R, Brockington M, Smith J, Talim B, Straub V, Robb S, Quinlivan R, Feng L, Jimenez-Mallebrera C, Mercuri E, Manzur AY, Kinali M, Torelli S, Brown SC, Sewry CA, Bushby K, Topaloglu H, North K, et al. (2007) Refining genotype phenotype correlations in muscular dystrophies with defective glycosylation of dystroglycan. Brain 130:2725-2735.

Grady RM, Zhou H, Cunningham JM, Henry MD, Campbell KP, Sanes JR (2000) Maturation and maintenance of the neuromuscular synapse: genetic evidence for roles of the dystrophin-glycoprotein complex. Neuron 25:279-293.

Grewal PK, Hewitt JE (2003) Glycosylation defects: a new mechanism for muscular dystrophy? Hum Mol Genet 12 [Suppl 2]:R259-R264.

Haines N, Seabrooke S, Stewart BA (2007) Dystroglycan and protein O-mannosyltransferases 1 and 2 are required to maintain integrity of Drosophila larval muscles. Mol Biol Cell 18:4721-4730.

Haliloglu G, Topaloglu H (2004) Glycosylation defects in muscular dystrophies. Curr Opin Neurol 17:521-527.

Ichimiya T, Manya H, Ohmae Y, Yoshida H, Takahashi K, Ueda R, Endo T, Nishihara S (2004) The twisted abdomen phenotype of Drosophila POMT1 and POMT2 mutants coincides with their heterophilic protein O-mannosyltransferase activity. J Biol Chem 279:42638-42647.

Jimenez-Mallebrera C, Torelli S, Brown SC, Feng L, Brockington M, Sewry CA, Beltran-Valero De Bernabe D, Muntoni F (2003) Profound skeletal muscle depletion of alpha-dystroglycan in Walker-Warburg syndrome. Eur J Paediatr Neurol 7:129-137.

Johnson KG, Tenney AP, Ghose A, Duckworth AM, Higashi ME, Parfitt K, Marcu O, Heslip TR, Marsh JL, Schwarz TL, Flanagan JG, Van Vactor D (2006) The HSPGs Syndecan and Dallylike bind the receptor phosphatase LAR and exert distinct effects on synaptic development. Neuron 49:517-531.

Jurado LA, Coloma A, Cruces J (1999) Identification of a human homolog of the Drosophila rotated abdomen gene (POMT1) encoding a putative protein O-mannosyl-transferase, and assignment to human chromosome 9q34.1. Genomics 58:171-180.

Kim DS, Hayashi YK, Matsumoto H, Ogawa M, Noguchi S, Murakami N, Sakuta R, Mochizuki M, Michele DE, Campbell KP, Nonaka I, Nishino I (2004) POMT1 mutation results in defective glycosylation and loss of laminin-binding activity in alpha-DG. Neurology 62:1009-1011.

Krasnow MA, Saffman EE, Kornfeld K, Hogness DS (1989) Transcriptional activation and repression by Ultrabithorax proteins in cultured Drosophila cells. Cell 57:1031-1043.

Lahey T, Gorczyca M, Jia XX, Budnik V (1994) The Drosophila tumor suppressor gene dlg is required for normal synaptic bouton structure. Neuron 13:823-835.

Lyalin D, Koles K, Roosendaal SD, Repnikova E, Van Wechel L, Panin VM (2006) The twisted gene encodes Drosophila protein O-mannosyltransferase 2 and genetically interacts with the rotated abdomen gene encoding Drosophila protein O-mannosyltransferase 1. Genetics 172:343-353.

Malinow R, Malenka RC (2002) AMPA receptor trafficking and synaptic plasticity. Annu Rev Neurosci 25:103-126.

Marrus SB, DiAntonio A (2004) Preferential localization of glutamate receptors opposite sites of high presynaptic release. Curr Biol 14:924-931.

Marrus SB, Portman SL, Allen MJ, Moffat KG, DiAntonio A (2004) Differential localization of glutamate receptor subunits at the Drosophila neuromuscular junction. J Neurosci 24:1406-1415.

Martin PT (2007) Congenital muscular dystrophies involving the O-mannose pathway. Curr Mol Med 7:417-425.

Martin-Blanco E, Garcia-Bellido A (1996) Mutations in the rotated abdomen locus affect muscle development and reveal an intrinsic asymmetry in Drosophila. Proc Natl Acad Sci USA 93:6048-6052.

McGee AW, Bredt DS (2003) Assembly and plasticity of the glutamatergic postsynaptic specialization. Curr Opin Neurobiol 13:111-118.
Michele DE, Barresi R, Kanagawa M, Saito F, Cohn RD, Satz JS, Dollar J, Nishino I, Kelley RI, Somer H, Straub V, Mathews KD, Moore SA, Campbell KP (2002) Post-translational disruption of dystroglycan-ligand interactions in congenital muscular dystrophies. Nature 418:417-422.

Pack-Chung E, Kurshan PT, Dickman DK, Schwarz TL (2007) A Drosophila kinesin required for synaptic bouton formation and synaptic vesicle transport. Nat Neurosci 10:980-989.

Petersen SA, Fetter RD, Noordermeer JN, Goodman CS, DiAntonio A (1997) Genetic analysis of glutamate receptors in Drosophila reveals a retrograde signal regulating presynaptic transmitter release. Neuron 19:1237-1248.

Qin G, Schwarz T, Kittel RJ, Schmid A, Rasse TM, Kappei D, Ponimaskin E, Heckmann M, Sigrist SJ (2005) Four different subunits are essential for expressing the synaptic glutamate receptor at neuromuscular junctions of Drosophila. J Neurosci 25:3209-3218.

Schneider M, Khalil AA, Poulton J, Castillejo-Lopez C, Egger-Adam D, Wodarz A, Deng WM, Baumgartner S (2006) Perlecan and Dystroglycan act at the basal side of the Drosophila follicular epithelium to maintain epithelial organization. Development 133:3805-3815.

Shcherbata HR, Yatsenko AS, Patterson L, Sood VD, Nudel U, Yaffe D, Baker D, Ruohola-Baker H (2007) Dissecting muscle and neuronal disorders in a Drosophila model of muscular dystrophy. EMBO J 26:481-493.

Sigrist SJ, Thiel PR, Reiff DF, Schuster CM (2002) The postsynaptic glutamate receptor subunit DGluR-IIA mediates long-term plasticity in Drosophila. J Neurosci 22:7362-7372.

Song W, Ranjan R, Dawson-Scully K, Bronk P, Marin L, Seroude L, Lin YJ, Nie Z, Atwood HL, Benzer S, Zinsmaier KE (2002) Presynaptic regulation of neurotransmission in Drosophila by the g protein-coupled receptor methuselah. Neuron 36:105-119.

Stewart BA, Atwood HL, Renger JJ, Wang J, Wu CF (1994) Improved stability of Drosophila larval neuromuscular preparations in haemolymphlike physiological solutions. J Comp Physiol A Neuroethol Sens Neural Behav Physiol 175:179-191.

van der Plas MC, Pilgram GS, Plomp JJ, de Jong A, Fradkin LG, Noordermeer JN (2006) Dystrophin is required for appropriate retrograde control of neurotransmitter release at the Drosophila neuromuscular junction. J Neurosci 26:333-344.

van der Plas MC, Pilgram GS, de Jong AW, Bansraj MR, Fradkin LG, Noordermeer JN (2007) Drosophila Dystrophin is required for integrity of the musculature. Mech Dev 124:617-630.

van Reeuwijk J, Janssen M, van den Elzen C, Beltran-Valero de Bernabe D, Sabatelli P, Merlini L, Boon M, Scheffer H, Brockington M, Muntoni F, Huynen MA, Verrips A, Walsh CA, Barth PG, Brunner HG, van Bokhoven H (2005) POMT2 mutations cause alpha-dystroglycan hypoglycosylation and Walker-Warburg syndrome. J Med Genet 42:907-912.

van Reeuwijk J, Maugenre S, van den Elzen C, Verrips A, Bertini E, Muntoni F, Merlini L, Scheffer H, Brunner HG, Guicheney P, van Bokhoven H (2006) The expanding phenotype of POMT1 mutations: from WalkerWarburg syndrome to congenital muscular dystrophy, microcephaly, and mental retardation. Hum Mutat 27:453-459.

Viquez NM, Li CR, Wairkar YP, DiAntonio A (2006) The B' protein phosphatase $2 \mathrm{~A}$ regulatory subunit well-rounded regulates synaptic growth and cytoskeletal stability at the Drosophila neuromuscular junction. J Neurosci 26:9293-9303.

Wagh DA, Rasse TM, Asan E, Hofbauer A, Schwenkert I, Durrbeck H, Buchner S, Dabauvalle MC, Schmidt M, Qin G, Wichmann C, Kittel R, Sigrist SJ, Buchner E (2006) Bruchpilot, a protein with homology to ELKS/ CAST, is required for structural integrity and function of synaptic active zones in Drosophila. Neuron 49:833-844.

Yao KM, White K (1994) Neural specificity of elav expression: defining a Drosophila promoter for directing expression to the nervous system. J Neurochem 63:41-51.

Zhang YQ, Bailey AM, Matthies HJ, Renden RB, Smith MA, Speese SD, Rubin GM, Broadie K (2001) Drosophila fragile X-related gene regulates the MAP1B homolog Futsch to control synaptic structure and function. Cell 107:591-603.

Zucker RS, Regehr WG (2002) Short-term synaptic plasticity. Annu Rev Physiol 64:355-405. 